\title{
On linking mechanism to invasive species impact
}

\author{
Marc Cadotte ${ }^{1}$ \\ ${ }^{1}$ University of Toronto-Scarborough
}

January 13, 2021

\begin{abstract}
Species invasion represents one of the major drivers of biodiversity change globally, yet there is widespread scientific and popular confusion and controversy about the nature of non-indigenous species (NIS) impact. This confusion stems from differing notions and understanding of what constitutes invasive species 'impact' and the scales at which it should be assessed. I argue that the proximate mechanisms determining invasive species impact happen at smaller scales where species interact, and by understanding these mechanisms, we can scale up to a broader understanding of how invasive species impact biodiversity. The mechanisms of NIS impact on potential competitors can be classified into four scenarios: 1) minimal impact from NIS inhabiting unique niche space; 2) neutral impact spread across the community and proportional to NIS abundance; 3) targeted impact on a small number of competitors with overlapping niches; and 4) pervasive impact that is disproportionate to NIS abundance and ostensibly caused by ecosystem modification that filters out other species. I develop a statistical test to distinguish these four mechanisms based on community rank-abundance curves. Using an example dataset from plant communities invaded by the dominant invasive vine, Vincetoxicum rossicum, I show that in long-term plots that had high native plant diversity and where V. rossicum increased, impact resulted in either targeted extirpations (scenario 3) or widespread biodiversity loss (scenario 4). Regardless of whether NIS impact is neutral, targeted or pervasive, the net outcome will be the homogenization of ecosystems and reduced biodiversity at larger scales, perhaps reducing ecosystem resilience.
\end{abstract}

\section{Hosted file}

Invasion_ImpactV3.pdf available at https://authorea.com/users/389274/articles/503897-onlinking-mechanism-to-invasive-species-impact 